\title{
Three-dimensional morphodynamics simulations of macropinocytic cups
}

\author{
Nen Saito ${ }^{1}$, Satoshi Sawai ${ }^{2,3,4}$ \\ 1 Universal Biological Institute, Graduate School of Science, University of Tokyo, \\ Bunkyo-ku, Tokyo, Japan \\ 2 Department of Basic Science, 3 Research Center for Complex Systems Biology, \\ Graduate School of Arts and Sciences, University of Tokyo, Meguro-ku, Tokyo, Japan \\ 4 Department of Biological Sciences, Graduate School of Science, University of \\ Tokyo, Bunkyo-ku, Tokyo, Japan
}

Email: cssawai@mail.ecc.u-tokyo.ac.jp or saito@ubi.s.u-tokyo.ac.jp

\section{- Abstract}

Macropinocytosis is non-specific uptake of the extracellular fluid playing ubiquitous roles in cell growth, immune-surveillance as well as virus entry. Despite its widespread occurrence, it remains unclear how its initial cup-shaped plasma membrane extensions forms without external physical support as in phagocytosis or curvature inducing proteins as in clathrin-mediated endocytosis. Here, by developing a novel computational framework that describes the coupling between bistable reaction-diffusion processes of active signaling patches and membrane deformation, we demonstrate that protrusive force localized to the edge of the patches can give rise to the self-enclosing cup structure without further assumption of local bending or contraction. Efficient uptake requires an appropriate balance between the patch size and the magnitude of protrusive force relative to the cortical tension. Furthermore, our model exhibits a variety of known morphology dynamics including cyclic cup formation, coexistence and competition between multiple cups and cup splitting indicating that these complex morphologies self-organize through mutually dependent dynamics between the reaction-diffusion process and membrane deformation. 

in which the extracellular fluid is taken up by internalization of micrometer-scale cupshaped membrane ruffles (Fig. 1A).

A wide range of cell types exhibits macropinocytosis either in a constitutive manner or under growth and other stimulating signals. Macropinocytosis is employed for nutrient uptake in Amoebae Dictyostelium (2) and certain cancer cells $(3,4)$. In immune cells, macropinocytosis plays a role in surveying foreign antigens (5-8). In neurons, macropinocytosis is also employed to regulate neurite outgrowth (9). Understanding the basis of these processes is of biomedical importance due to its link in tumor growth $(3,4)$, virus entry $(10)$ and spread of prions related to neurodegenerative disease (11). Despite wide occurrence of these phenomena, however, the basic question regarding the very nature of the membrane deformation remains unanswered. The large-scale cup formation involves complex spatiotemporal regulations of signaling molecules and cytoskeletal machineries. Unlike the better-studied clathrin-coated pits, where membrane invagination of $\sim 100 \mathrm{~nm}$ diameter is formed by clathrin assembly, macropinosome have no apparent coat structures and their size varies between $0.2-5 \mu \mathrm{m}$ in diameter $(6,12,13)$. Furthermore, in contrast to phagocytic cup which extends along the extracellular particles $(14,15)$, there is no such support to guide the macropinocytic cups externally. These morphological and dynamical features distinct from other endocytic processes indicate a mechanism unique to macropinocytosis that remains to be elucidated.

The initial stage of cup formation is identifiable by formation and expansion of an active signaling patch in the plasma membrane that consists of intense accumulation of phosphatydilinositol $(3,4,5)$ tris-phosphate (PIP3) and the active form of small GTPases such as Ras, Rap and Rac surrounded by an edge region enriched in F-actin, Arp2/3 and the Scar/WAVE complex $(13,16)$. For brevity, we shall refer to this region as 'active patch'. Relative positioning of these factors remains affixed as the patches grow in size (Fig. $1 A$ left). The edge of the active patches protrude outward up to several micrometers thus forming the rim of a cup which then curves inward to ingest extracellular fluid (Fig.

$621 A$ middle). The resulting cup closes by membrane fusion to form a macropinosome (Fig. $631 A$ right) which further matures and fuse with lysosomes for degradation of incorporated 64 extracellular solutes (16). The active patch is thought to self-organize by combination 65 of autocatalytic activation of Ras and PIP3 production and their diffusion (17-20). 
When observed in the ventral membrane along the substrate, active patches appear as traveling spots and waves - a hallmark of reaction-diffusion mediated pattern formation $(17,18,21-23)$. Although these active patches appear to act as a prepattern or 'template' for macropinocytic cup (24), little is known how these materialize into the formation of the cup itself.

In recent years, progress in theoretical and computational approaches have allowed one to address dynamical properties of cellular- and sub-cellular scale membrane deformation such as amoeboid motion, filopodia formation. Common to these modeling approaches is mathematical formulation that describes the underlying regulatory kinetics together with a moving boundary. This physico-chemical coupling makes the problem unique and challenging, since the very nature of highly deformable boundary requires elaborate techniques to solve the interface physics that are often computationally laborious and expensive. Many of the studies have focused on cases that can be approximated in oneand two-dimensional space including but not limited to formation of filopodia during axonal elongation (25), pseudopodium in ameboid migration (26), lamellipodia of fish keratocytes (27-29), while relatively few attempts have been made for 3 dimensional dynamics (30-32). Models of 2-D dynamics by the active patches constrained to the ventral (18) or the dorsal-side (19) of the plasma membrane has been analyzed. Given its geometry, understanding the full nature of membrane deformation in macropinocytosis poses a challenge that requires full 3-dimensinal modeling with topological changes in membrane. In this paper, we propose and analyze a minimalistic 3-D model to address the relationship between the self-organizing active patches and the geometry of macropinocytic cup formation and closure. Our results indicate that relative simple rule of self-organization coupled with membrane protrusion can explain the entire sequence of the dynamics starting from patch expansion, cup formation to cup closure without further need for specialized machineries to regulate local curvature.

\section{Model}

96 We adopt a modeling strategy that combines two elementary processes: (i) deformation 97 of the membrane and (ii) reaction-diffusion process of signaling molecules on the 98 deformable membrane. To describe the membrane, we employ the phase-field method, 
99 which allows one to simulate interfaces with complex geometry such as growing crystals

$100(33,34)$, vesicle coarsening or fission (35) as well as an overall shape of migratory cells

$101(18,20,25,27)$. The phase-field approach allows one to compute cellular membrane

102 deformation on the order of micrometers in spatial scale and seconds to minutes in

103 timescales, which is in contrast to nanometer-scale models that describe microsecond

104 order phenomena (36). Here, an abstract field variable $\phi$ is introduced to describe the

105 cell interior region $\phi=1$ and the exterior region and $\phi=0$ (Fig. $1 B$ ). $\phi$ is assumed

106 to be continuous and varies sharply at the interface with finite width characterized by a

107 small parameter $\epsilon$. Following previous studies $(18,27)$, here, we adopt the following 108 equations (see SI Text for derivation)

$$
\tau \frac{\partial \phi}{\partial t}=\eta\left(\nabla^{2} \phi-\frac{G^{\prime}(\phi)}{\epsilon^{2}}\right)-M_{V}\left(V-V_{0}\right)|\nabla \phi|+F_{\text {poly }}|\nabla \phi|,
$$

111 where $G^{\prime}=16 \phi(1-\phi)(1-2 \phi)$ and $V=\int \phi d r$. The first term in the right hand side

112 represents curvature-driven force associated with surface tension $\eta$. The second term 113 imposes a constraint on the cell volume to $V_{0}=4 \pi R_{0}^{3} / 3$ where $R_{0}$ is the cell radius and

$114 M_{V}$ is a constraint parameter. The third term describes the force normal to the interface 115 driven by dendritic actin polymerization. The magnitude of force $F_{\text {poly }}$ is assumed to 116 be a function of the local concentrations of signaling molecules as described below.

118 For time development of the signaling molecule, let us assume an interconversion 119 between the active form $A$ on the plasma membrane and inactive cytosolic form $B$ : $120 A \leftrightarrows B$ (Fig. $1 C$ ). The total number of molecules is fixed to $A_{\mathrm{t}}$, and the conversion 121 from $A$ to $B$ is assumed to take place at a constant rate, whereas that of $B$ to $A$ is 122 facilitated in an autocatalytic manner. This scheme gives rise to bi-stability, where $A$ 123 takes two states: zero and a finite positive value. When $A$ is locally perturbed from $124 A=0$, a domain where increase in $A$ takes place spreads in space and eventually stops 125 due to depletion of $B$, thereby creating a stable spot pattern which we shall consider as a 126 mathematical representation of an active patch. Let us further introduce a factor $I$ that 127 inhibits conversion of $B$ to $A$ so that the active patch has a finite lifetime. The above 128 basic reactions are expressed in the following dimensionless reaction-diffusion equations 129 (see SI Text for derivation): 


$$
\frac{\partial A}{\partial t}=\frac{A^{2} B}{1+A^{2} / \alpha^{2}} \frac{1}{1+I}-A+D_{A} \nabla^{2} A
$$

(2)

$$
\frac{\partial I}{\partial t}=k_{1} A^{2}-k_{2} I+D_{I} \nabla^{2} I
$$

where $D_{A}$ and $D_{I}$ are diffusion constants of $A$ and $I$ molecules, respectively. The parameter $\alpha$ dictates a half saturation concentration of the Hill function in the autocatalytic reaction $B \rightarrow A$. The second equation assumes a negative feedback that produces the inhibitor $I$ at the rate $k_{1} A^{2}$ and degraded at a constant rate $k_{2}$. When diffusion of $B$ molecule is sufficiently fast, $B=A_{\mathrm{t}} / S-\langle A\rangle$, where $S$ is the cell surface area $S=\int \psi / \varepsilon \mathrm{dr}^{3}$ and $\langle A\rangle$ is the total of $A$ divided by $S$. Note that ' $A$ ' and ' $B$ ' can also be membrane-bound factors as long as diffusion of ' $B$ ' is sufficiently fast compared to that of ' $A$ '. For $I=0$ and $k_{1}=0$, the reaction-diffusion equations are reduced to the well-studied wave pinning model of cell polarization $(37,38)$.

144 To define spatial coordinates occupied by the plasma membrane, let us introduce an auxiliary phase-field $\psi=\left(1+e^{-\beta(\phi(1-\phi)-\theta)}\right)^{-1}$ which specifies the interface between cell exterior $(\phi=0)$ and interior $(\phi=1)$ region. $\psi$ takes constant value $\psi=1$ at the cell membrane and $\psi=0$ elsewhere (Fig. 1B). Here, $\beta$ takes a sufficiently large value so as to render the interface between inside and outside of the membrane sharp. $\theta$ is set so that the $\psi$ is non-zero at the interface of $\phi$ with thickness $\epsilon$. The unique aspect of the present approach is the introduction of this auxiliary field $\psi$ thereby allowing Eqs. (2) and (3)) to be solved numerically at the interface only. In contrast, previous 2D models $(18,27,28)$ made distinction only between occupied (cell; $\phi=1$ ) and vacant (no-cell; $\phi=0$ ) regions and assumed reaction that take place throughout the occupied space. Using $\psi$, we arrive at the following equations:

$$
\frac{\partial}{\partial t} \psi A=-\nabla \cdot(\psi A v)+\psi\left[\frac{A^{2} B}{1+A^{2} / \alpha^{2}} \frac{1}{1+I}-A\right]+D_{A} \nabla(\psi \nabla A)
$$

$$
\frac{\partial}{\partial t} \psi I=-\nabla \cdot(\psi I v)+\psi\left[k_{1} A^{2}-k_{2} I\right]+D_{I} \nabla(\psi \nabla I)
$$


160 where the first terms in the right hand side are the advection term and $\boldsymbol{v}$ is given by

$$
\boldsymbol{v}=-\left[\frac{\eta\left(\nabla^{2} \phi-\frac{G^{\prime}(\phi)}{\epsilon^{2}}\right)}{|\nabla \phi|}-M_{V}\left(V-V_{0}\right)+F_{\text {poly }}\right] \frac{\nabla \phi}{|\nabla \phi|} .
$$

Given that protrusive actin filaments are concentrated at the edge of the activated patch $(16,24)$, we assume that protrusion is facilitated when $A$ is within a certain range as illustrated in Fig. 1D. To implement this, the actin-dependent force generation in Eq. (1) and (6) are given as in the form of the force term

$$
F_{\text {poly }}(A(\boldsymbol{r}))=F \frac{\left(A / K_{1}\right)^{n_{h}}}{1+\left(A / K_{1}\right)^{n_{h}}} \frac{1}{1+\left(A^{2} / K_{2}\right)^{n_{h}}}
$$

\section{- Results}

Mutually dependency between the patch dynamics and deformation drives cup

formation and closure. First, we demonstrate an overall time development of the 3dimensional model in the absence of the inhibitor by setting $k_{1}=0$. As an initial condition, we chose a membrane sphere with $A=I=0$ except for a small circular region with radius $r_{\text {init }}$ where the local concentration of $A$ takes random value from 0 to 5.0 on each grid. Representative results are shown in Fig. $2 A$ (see also Movie S1). Due to bistability, a local active patch defined by high $A$ begin to invade the basal state of low $A$ as a propagating front (Fig. $2 A t=4$ orange region) . As the patch expanded, the membrane protruded at the patch periphery and formed a cup shaped circular extension (Fig. $2 A ; t=4-24$ orange and green border). After the patch grew to a certain size, the expansion slowed down. At the same time, the protrusion formed an overhang while the center of the patch curved slightly inward to form a cup (Fig. $2 A ; t=24-44$ ).

187 The rim of the cup shrunk and annihilated as the membrane sealed itself to completely 188 surround a large volume of extracellular space (Fig. $2 A ; t=65$ ). The coordinated manner in which a circular ruffle encircling a non-protruding area extended, shrunk and 
190 closed showed a close parallel to the cup dynamics observed in Dictyostelium (24). Also

191 of note is the marked accumulation of $A$ in the inner territory and its exclusion from the

192 rim which are in good agreement with the patterns of bona fide active patch marker $\mathrm{PIP}_{3}$

193 and Ras-GTP $(24,39-41)$.

195 Whether the cup closed or not depended on the parameters and the initial condition. Cup 196 closure was judged by evaluating whether the region with $\phi=0$ surrounded by $\phi=1$ 197 based on quasi 3-dimensional simulations where dimensionality was reduced in an axis198 symmetric coordinate for easier detection of morphology criteria and computation 199 involving exhaustive parameter search (fig. S1 $A-H$; see also Methods). In the phase field 200 framework, the topological change that accompanies membrane fusion is naturally established by simply solving the partial differential equation Eq. (1) without additional numerical implementation. For enclosure of a large volume, the critical parameters were the ratio between force per unit area $F$ and the surface tension $\eta$ i.e. $F / \eta$, and the total amount of $A$ and $B$ per unit area $a_{\mathrm{t}} \equiv A_{\mathrm{t}} / 4 \pi R_{0}^{2}$. Figure $2 B$ illustrates a phasediagram for cup closure. Black regions represent parameters that were unable to support enclosure, otherwise color represents the enclosed volume relative to the cell volume $V_{0}$

207 (Fig. 2B). A similar portrayal of the parameter space was obtained based on the elapsed 208 time between the patch initiation and the cup closure (fig. S2A) and the ingestion efficiency (fig. S2B). All phase diagrams were obtained by averaging results from two

210 initial patch size $r_{\text {init }}$ (fig. S2C,D). The parameter space could be divided into four 211 domains: Phase I - IV based on the success rate of closure. Phase I includes the example

212 shown in Figure $1 A$ where parameters supported enclosure in all cases. Phase II consists 213 of parameters where cup closure depended on the initial conditions. Here, due to small 214 patch size, the cup and hence the enclosed extracellular volume was sometimes extremely 215 small (Fig. 2C). In Phase IV, cup closure failed for all simulations runs (Fig. 2D, E).

217 Requirements for successful cup formation and closure (Phase I) can be understood from 218 the characteristic dynamics observed when cups failed to support large volume uptake. 219 Phase IV consisted of two patterns of incomplete closure depending on the value of $F / \eta$. 220 When $F / \eta$ was small, patches and cups persisted indefinitely without shrinking or 221 closing (Fig. 2D) for both high and low $a_{\mathrm{t}}$. At high $F / \eta$, cup shrunk without closing 222 when $a_{\mathrm{t}}$ was not high (Fig. $2 E$ ). The behavior at low $F / \eta$ was due to lack of 
223 sufficient protrusive force for cup development. Consider a cross section of a protrusion 224 with width $2 R$ (fig. S3), the force per unit length $F$ exerted on the semicircular head of 225 length $l=\pi R$ should be twice as large as the line tension $\eta$ required to maintain the 226 protrusion. Hence, the minimal force $F^{*}$ must obey $F^{*} / \eta=1 / R=\pi / l$. Based on 227 cortical tension of $\sim 0.7 \mathrm{nN} / \mu \mathrm{m}$ (42) and an estimate for protrusive force $\sim 6.5$ to 9 $228 \mathrm{nN} / \mu \mathrm{m}^{2}$ (protrusive force by a single microfilament 5 to $7 \mathrm{pN}$ times the filament density 229 (43)), the condition $F / \eta>1 / R$ is satisfied for protrusion width of $\gtrsim 0.2 \mu \mathrm{m}$. While $230 l$ in real cells has not been measured quantitatively, projections thinner than $0.2 \mu \mathrm{m}$ 231 would require larger $F / \eta$ than the above estimate. Relative ease of imaging the cups under the conventional confocal microscope suggests they are above the diffraction limit $(>0.25 \mu \mathrm{m})$ which is within this force requirement. Because the spatial resolution of our numerical simulations were limited by the computational time, for systematic parameter studies, parameters $K_{1}$ and $K_{2}$ in Eq. (7) were chosen so that $l \sim 1.5 \mu \mathrm{m}$ (Fig. $1 D$, black plateau; fig. S3), hence $F^{*} / \eta \sim 2.0 \mu \mathrm{m}^{-1}$ which is consistent with the boundary in the phase diagram (Fig. $2 B$; red dashed line). In contrast to the force constraints at small $F / \eta$, the characteristic behavior at high $F / \eta$ (Fig. $2 E$ ) was due to lack of sufficient patch size at small $a_{\mathrm{t}}$. Here, the resulting small cups gave rise to high negative curvature which in turn provides strong restoring force in the inner territory that prevented the protrusion from curling inward. This resulted in a shmoo-like cell

242 morphology (Fig. $2 E, t=70$ ) which eventually returned to symmetric sphere as the patch

243 disappeared. This patch attenuation was a distinct feature that arose due to self244 consistency requirement that the edge of the patch must define the point of protrusion and vice-versa. If protrusion were to come close and coalesce due to high tension, the region that it surrounded must also disappear. One should note that the same parameters support

247 a persistent patch if it were not for deformation, thus the coupling of reaction-diffusion 248 process and deformation is essential.

250 In Phase III, cup formation was observed to repeat at the same site. A similar behavior 251 has been observed in the standard axenic strain of Dictyostelium discoideum and in an 252 even more pronounced form in the null-mutant of RapGEF ( $g f l B)$ (44). In our 253 simulations, there were two patterns of repetition both of which occurred under conditions 254 that allowed formation of exceedingly large cup (Figs. $2 F$ and $G$ ). In the first examples 255 (Fig. $2 F$; see also Movie S2), cup closed at its waist (Fig. $2 F ; t=240 \mathrm{sec}$ ) while the 
256 remaining open half continued to expand at the edge (Fig. $2 F ; t=356$ ). After the second

257 closure (Fig. $2 F ; t=492$ ), the rim disappeared and there was no more cup formation.

258 The other pattern occurred for slightly weaker force (Fig. 2G; see also Movie S3). Here,

259 cup closure was stalled in the middle (Fig. $2 G ; t=296$ ) as the patch continued to expand

260 laterally before the next attempt at the cup-formation (Fig. $2 G ; t=400$ ). While

261 distinction between these two behaviors is difficult to resolve experimentally, the

262 markedly elongated cell shape (Fig. $2 F ; t=492$ and Fig. $2 G ; t=480$ ), and the lengthening

263 of time required for enclosure (fig. S2A) are in accordance with what has been reported

264 for the $g f l B$ mutant. The size of the Phase III region depended on the time scale of

265 deformation $\tau$. In the examples shown above $(\tau=10 \mathrm{sec})$, normal cup closure (Phase

266 I) was predominantly observed, and Phase III was confined to a narrow domain between

267 Phase I and IV (Fig. 2B). For smaller $\tau(\tau=5 \mathrm{sec})$, Phase II became dominant, and

268 Phase I and III were both confined to narrow regions in the parameter space (fig. S4A).

269 In contrast, at large $\tau(=20 \mathrm{sec})$, the Phase I and III regions expanded (fig. S4B).

270 Overall, normal cup closure (Phase I) is realizable at large $\tau$, however it comes at the cost

271 of also inviting repetitive dynamics that are often incomplete (Phase III) in addition to

272 the overall process slowing down (fig. S4B middle) making the process less efficient (fig.

$273 \mathrm{~S} 4 B$ right).

Inhibitor and mass conservation determines duration of the patch and cup dynamics.

277 Large cell-size cups are frequently observed in the axenic strain of Dictyostelium (24, 40,

278 45), however they do not exist indefinitely. The active signaling patches are mostly

279 transient and eventually vanishes with a lifetime of few minutes $(17,18,22,23,46)$. In

280 our simulations, the active patches on their own have finite lifetime when the presence of

281 the inhibitor $I$ is non-negligible $\left(k_{1} \neq 0\right)$. For $k_{1}=k_{2}=2.0 \times 10^{-4}$, the inhibitor $I$

282 increases at a much slower timescale than the initial expansion of the active patch.

283 Eventually, $I$ becomes high enough to suppress $A$ i.e. the activate patch (fig. S5) when

$284 a_{\mathrm{t}}$ satisfies a certain condition (see SI Text). In Phase III, the presence of the inhibitor

285 repressed the repetitive cup formation and abolished the ruffle formation (fig. S5C and

$286 \quad F$ ), whereas no change was observed for Phase I and II. 
288 Besides the inhibitor, the assumed mass conservation of the signaling molecule can also

289 prevent futile formation of excessively large cup. This effect becomes most evident

290 when there are simultaneous and constitutive occurrence of active patches. Let us

291 examine slightly complex situations where activation of $A$ is allowed to occur at random

292 positions $x_{\mathrm{c}}$ at rate $\theta$ per volume. The spatial profile of noise follows $\mathcal{N}(\boldsymbol{x})=$

$293 \mathcal{N}_{0} \times \exp \left(-\frac{\left|x-x_{c}\right|^{2}}{2 d^{2}}\right)$, where $d$ is the initial nucleation size, and $\mathcal{N}_{0}$ is the noise

294 intensity that follows an exponential distribution with the average $\sigma$. Figure $3 A$ shows

295 representative snapshots from independent simulation runs (see also Movie S4). A new

296 active patch was nucleated before existing cups closed thus allowing multiple cups to

297 coexist. Depending on the size and amplitude of the noise, some cups closed successfully,

298 while others shrunk and vanished before they can close. Incomplete closure occurred

299 even when the same parameter supported closure for an isolated cup (Phase I). This

300 can be explained by effective lowering of $a_{\mathrm{t}}$ available per cup. Due to continual cup

301 formation and closure, the cell shape deviated markedly from the initial sphere and took

302 complex and processive morphology that highly resembled axenic strains of

303 Dictyostelium. In the parameter regime that supported relative large and slow cup

304 closure (Phase III), these features became more exaggerated (Fig. 3B). Multiple cups

305 were indeed frequently observed in Dictyostelim cells, and they either successfully closed

306 to form endosomes or vanished without closing (40).

307

308 Excitability arises in the presence of strong inhibitory signal and drives cup splitting

309 dynamics. The cup dynamics described above was monotonous, meaning that the

310 initial active patch more or less dictated when and where a cup formed, and it grew due

311 to bistability until it consumed all $B$. In Dictyostelium, however, cups are known to

312 also multiply or reduce in number by splitting and coalescence of existing cups (24). In

313 the present model, when the production of $A$ is no longer a saturating function (large $\alpha$

314 in Eq. 2), the active patch (a region with high $A$ ) can become out of phase with a high $I$

315 region. As a consequence, the region occupied by high $I$ will trail behind a moving

316 active patch and can disrupt it (fig. S6A, B). To study this behavior in detail, let us

317 consider a case $\alpha \rightarrow \infty$ so that Eq.(2) now becomes

318

$$
\frac{\partial A}{\partial t}=\frac{A^{2} B}{1+I}-A+D_{A} \nabla^{2} A
$$


$319 \quad(8)$

320 which is the same equation introduced earlier as a part of a model for the patch dynamics

321 in circular dorsal ruffle (19). The key difference in the present model, apart from

322 incorporation of the membrane deformation, is that Eq. (8) is coupled to Eq. (3) with

323 quadratic dependence on $A$ which is essential for providing a rich behavior as follows.

324 The equation has three different parameter regimes: mono-stable, bi-stable, and excitable

325 (Fig. $4 A$, see also fig. S7A-C for finite $\alpha$ ). In the excitable regime, null-cline analysis

326 (Fig. $4 B$, left panel) shows that, for a small $\langle A\rangle$ (i.e., for $B>2 \sqrt{k_{1} / k_{2}}$ ), small

327 perturbation from the fixed point $A=0$ gives rise to a large excitation of $A$. For large

$328\langle A\rangle$ (i.e., for $B<2 \sqrt{k_{1} / k_{2}}$ : right panel in Fig. $4 A$ ), excitability disappears and $A$ falls immediately to the basal state even when strongly perturbed. Interestingly, this in turn brings the system back to an excitable state hence $A$ is again easily perturbed and brought transiently to a high level. In other words, depending on $\langle A\rangle$ i.e. the total size of active patches, excitability is switched on and off in a sequential manner. This switching of excitability destabilizes the expanding front of active patches (fig. S6A), similar to splitting patches or waves observed in the ventral side of the plasma membrane $(17,18,21-23)$.

337 When coupled to membrane deformation, however, a broad protrusive force profile in the region surround by a patch (fig. S6C and $D ; t=8,0<r<3$ ) smoothed out the fragmented active patches before daughter cups developed (fig. S6E and $F$ ). While this can be circumvented at small $F$, fragmented cups then failed to close due to lack of sufficient protrusion (fig. S6G). A recent CryoEM study of the ventral actin waves demonstrated that the form and alignment of actin filaments at the edge of the patch and those in the inner region are distinct and thus hints at the presence of debranching factors that trails

344 behind the expanding edge (47). Such notion is line with sharp localization of 345 Scar/WAVE complex at the edge of a patch (24) and depolymerization factor Coronin at 346 the rear of the edge (Bretschneider et al 2009. Biophys J). To study such an effect in 347 the model, let us modify the force term so that that $I$ not only suppresses amplification of $348 A$ but also competitively inhibits force generation by $A$, so that

$$
F_{\text {poly }}(A(\boldsymbol{r}), I(\boldsymbol{r}))=F \frac{\left(A / K_{1}\right)^{n_{h}}}{1+\left(A / K_{1}\right)^{n} h} \frac{1}{1+\left(I / K_{2}\right)^{n_{h}}}
$$


350 Note that the original form of $F_{\text {poly }}$ (Eq. 7) is recovered when $I$ is at the steady state;

351 i.e. $\dot{I}=0$, and $D_{I}$ is negligible. The periphery of the active patch is defined by high

$352 A$ and low $I$, thus under Eq. (9), the force profile became restricted to the edge (fig. S6H,

$353 I ; t=8)$. Protruding force in the inner territory only appeared later to surround the split

354 patches (fig. $\mathrm{S} 6 H ; t=15$ ). Accordingly, expanding active patches broke up repeatedly

355 while some of the daughter patches quickly merged with existing ones and gave rise to 356 cup-shaped circular ruffles (Fig. $4 D ; t=20,28$, and Movie S5). By ruffles, we mean

357 that the rim of cup was no longer smooth and circular but more undulated and complex

358 in shape. Splitting of an activate patch during ruffle formation causes its fragmentation

359 (Fig. $4 D ; t=36$ ). A notable difference from multiple cups occurring in non-excitable

360 regime (Fig. 3) was that multiple patches and cups continued to emerge starting from a

361 single founder. These sequence of events and their appearance; splitting followed by

362

363

364

365

366

367

368

369

370

371

372

373

374

375

376

377

378

379

380

381

382 formation of cup-shaped ruffles (Fig. 2D) are remarkably similar to how, in Dictyostelium, an active Rac and F-actin rich region expands together with membrane ruffles then become fragmented into multiple macropinocytic cups (24).

\section{Discussion}

The present work suggests an unexpectedly simple yet concerted mechanism that underlies formation and closure of macropinocytic cups. First, a locally activated signaling patch represented by high $A$ in the model appears. The active patch expands in a self-organized manner via autocatalytic transition of bistable nature from the state of low $A$ to high $A$. From there, two key assumptions in the model dictate the fate of the patch and the resulting cup. (i) Growth of an activated patch is limited due to the finite amount of the signaling molecules (Eq. (2)); i.e. the sum of $A$ and $B$ molecules $A_{\mathrm{t}}$ (or $a_{\mathrm{t}}$ in the normalized form) is fixed within a cell. (ii) Protruding force is restricted to the edge of an active patch (Eq. (7) and (9)) $(16,48)$. Due to the constraint (i), a patch first expands (fig. S8; $t=t_{1}$ ), then slows down as it reaches its size limit (fig. S8; $t=t_{2}$ ). The edge continues to protrude and forces the patch area to expand. However because $B$ is no longer available, $A$ at the patch boundary must be brought down to the low state. Thus the position of the patch boundary (fig. S8; $t=t_{3}$, black circle) is effectively displaced from the rim of a cup (fig. S8; $t=t_{3}$, blue asterisk) towards the inner territory (fig. S8; $t=t_{3}$ ). Because protrusive force is generated at the patch boundary (ii), the 
383 protrusion begins to curve inward, forming an overhang (fig. S8; $t=t_{4}, t_{5}$ ) and continues

384 to advance until they meet each other. We should note that spontaneous curvature is 385 assumed to be negligible in the present formulation (Eq. (1)), and that the involution arises

386 due to mutuality between the reaction-diffusion process and deformation dynamics in

387 defining the position of the protrusion. In this light, the work brings to light a distinct

388 mechanism of membrane invagination that contrasts with those driven by local curvature;

389 e.g. formation of endocytic vesicles by clathrin (49) and BAR-domain containing proteins

$390(50,51)$.

391

392 The high similarity between the range of complex morphology dynamics observed in the present simulations and those in Dictyostelium cells suggest that the kinetics adopted in the current model captured the essence of the underlying regulation and the cell mechanics. The critical parameter that determined the occurrence of a patch and its size was $a_{\mathrm{t}}$. Strong candidates for $A$ and $B$ are active and inactive form of small GTPase such as Ras, Rap and Rac or their upstream and downstream signaling partners such as PI3K which are all found enriched in the activated patch $(24,39,52)$. PI3kinase requires Ras binding for its activity $(53,54)$ and thus the variable $A$ may represent Ras in complex with PI3kinase or its product PIP3 and the variable $B$ may be regarded as their inactive forms. In fibroblasts, microinjection of active Ras protein induces macropinocytosis (55). RasS mutation in Dictyostelium cells are known to inhibit macropinocytosis $(39,56)$. These perturbations can be understood from increasing or decreasing $a_{\mathrm{t}}$ and hence the size of the active patch. Due to non-dimensionalization in Eq. (2), lowering of $a_{\mathrm{t}}$ can also result from decrease in the autocatalyic reaction $B \rightarrow$ $A$ (see SI Text). The analysis is in line with a recent suggestion based on the observation of smaller patches and macropinosomes in PI3kinase mutants $(24,57)$ and in a double mutant of Akt/PkbA and PkbR1 (57) that there likely is a positive feedback loop between PIP3 production and its downstream PKB in Dictyostelium (57).

411 Apart from bi-stability, our model suggests that excitability arises when the self412 amplification of $A$ is less saturated (i.e., large $\alpha$ ). In Dictyostelium, loss of Ras 413 GTPase-activating protein (RasGAP) Neurofibromin (NF1) causes formation of 414 oversized macropinosomes, increases fluid uptake and facilitates cell growth in liquid 415 media (45). In our model, a decrease in RasGAP would correspond to lowering the 
416 rate of reaction $A \rightarrow B$. Due to parameter non-dimensionalization in Eq. (2), not only

$417 a_{\mathrm{t}}$ but also $\alpha$ increases in this case (see SI Text). Since elevation in $\alpha$ brings the

418 system to the excitable regime, attenuation of RasGAP makes it an ideal point of

419 perturbation to enhance macropinocytosis; i.e. an increase in the number of patches due

420 to splitting in addition to supporting a larger patch size. Both expression of activated

421 Ras in the wild-type cell (41) and Ras-GAP mutation (45) are known to enhance fluid

422 uptake. Our model assumes that the inhibitor $I$ weakens autoregulatory amplification

423 of $A$. From $\dot{I}=0$ at Eq. (3), one can see that $I$ imposes saturation in the production

424 of $A$ even at high $\alpha$, and thus has a similar effect to changing $\alpha$. In addition, $I$ acts

425 critically for patch duration as well as for cup splitting (Fig. 4D). For large $a_{\mathrm{t}}$, absence

426 of the inhibitor $I$ caused cup formation to repeat at the same site due to incomplete

427 closure of an oversized cup (Fig. 2B; Phase III). Following the line of thoughts that $A$

428 maybe regarded as an activated form of small GTPase, $I$ would be a factor that

429 suppresses guanine nucleotide exchange factor (GEF)s. In line with the model behavior,

430 a knockout of Ras/Rap GEF (GflB) does indeed repeat cup formation at the same site

431 (44).

432

433 For efficient uptake, the key mechanical parameter was the magnitude of protrusive force

434 relative to that of the cortical tension. Our model predicts that high $F / \eta$ should facilitate

435 macropinocytosis, which is in line with increase in macropinocytosis under decreased

436 membrane tension (58). Although the variable $A$ and $I$ are abstract and collective

437 representation of regulatory factors, from the cell mechanics point of view, they must be

438 closely linked to the nucleator Arp2/3 complex bound to the polymerizing actin (19), and

439 a debranching factor such as coronin in complex with F- actin, respectively. Mass

440 conservation of $A+B$ in the model could hence be attributed to competition for limited

441 supply of actin or nucleating factors (59-64). In line with the global constraint,

442 macropinocytic cup formation is known to compete with pseudopod formation (40).

443 Appearance of an active patch on one side of the plasma membrane excludes another

444 patch from appearing on other locations (65). As for the variable $I$, its simulated profile

445 (fig. S6B) is in line with that of coronin which trails behind the traveling actin waves (66).

446 Apart from the role of $I$ to inhibit amplification of $A$ (Eq. 2), our model assumed $I$ to

447 increase by duplex of $A$ (Eq. 3 ). In this regard, $I$ can be regarded as a complex of 448 coronin cross-linked with actin filaments $(67,68)$. In the activated patch, coronin may 
449 mediate the switch in the orientation of the dendritic actin filaments from those facing the

450 membrane to those that are parallel (47). Such change would lead to vanishing force in

451 the direction normal to the membrane interface consistent with our assumption that the

452 force generation by $A$ is competitively attenuated by $I$ (Eq. (9)).

453

454 The current framework should be applicable to other related form of membrane 455 deformation. Dendritic cells exhibit numerous multi-layered membrane ruffles and 456 macropinosomes_(69, 70). Such coexistence of multiple internalized vesicles was rarely 457 observed in the present simulation due to minimization of the global surface area assumed 458 in the Allen-Cahn type phase-field equation (Eq. 1). Further exploration in the parameter 459 space, specifically for large $a_{\mathrm{t}}$ and $\alpha$, with perhaps additional implementation to control 460 the speed of the vanishing vesicles may uncover related morphological features. In some 461 cancer cells, the dorsal side of the plasma membrane is covered by circular membrane 462 ruffle associated with macropinocytosis $(19,22)$. This so-called "circular dorsal ruffles" 463 (CDR) is initiated from a F-actin-rich circular projections on the dorsal cell surface. 464 Similar to the present simulations, the ring region expands then contracts, then forms a 465 cup-like structure. Restriction of the dynamics in the dorsal side can be explained in the 466 presence of dorsal-ventral asymmetry in the parameter at Phase I (e.g., Fig. $2 A$ ). We 467 should note, however, that because multiple macropinosomes can form within a single 468 cup (19), there likely is an additional mechanism at play to form these smaller ruffles. 469 Further extension of the model such as to incorporate local change in tension $\eta$, which 470 likely depends on localized myosin $I(71,72)$ may help explain these dynamics. Spatial 471 restriction of the patch-driven dynamics may also help explain ruffling with a linear 472 geometry known in macrophages where membrane ruffles many near the cell edge fold 473 back on itself to close the cup $(6,73)$. Spatially much finer filopodial projections that 474 resemble a tent-pole are also known (8). Future work should address the relation 475 between these distinct subcellular morphologies and the basic cup dynamics uncovered 476 in this work. 
480 Time evolution of equation for $\phi, A$ and $I$ was numerically solved using the standard

481 explicit Euler method with mesh size $d x=0.1 \mu \mathrm{m}$ and $d t=4.0 \times 10^{-4} \mathrm{sec}$. For $A$

482 and $I$, instead of solving Eqs.(4) and (5) directly, we computed the following equations

483

484

$$
\frac{\partial A}{\partial t}=-\nabla \cdot(A \vec{v})+D_{A} \beta(1-\psi)(1-2 \phi) \nabla \phi \nabla A+D_{A} \nabla^{2} A+\frac{A^{2} B}{1+A^{2} / \alpha^{2}} \frac{1}{1+I}-A
$$

$$
\frac{\partial I}{\partial t}=\quad-\nabla \cdot(I \vec{v})+D_{I} \beta(1-\psi)(1-2 \phi) \nabla \phi \nabla I+D_{I} \nabla^{2} I+k_{1} A^{2}-k_{2} I,
$$

which derives from the relation $\psi=\left(1+e^{-\beta(\phi(1-\phi)-\theta)}\right)^{-1}$. The above equations were solved on all lattice sites above the cut-off threshold $\psi>10^{-3}$, otherwise $A$ and $I$ were allowed to simply decay at a rate $\gamma_{2}=10.0\left[\mathrm{sec}^{-1}\right]$. Likewise, the equation for $\vec{v}$ in Eq.(6) is computed for all sites $|\nabla \phi|>10^{-3}$, otherwise $\vec{v}=0$. Note that, immediately after the cup closure, the internalized cup shrinks and eventually vanishes due to the surface tension which causes numerical instability due to an abrupt increase in $A$ on the shrinking membrane. To avoid this instability, an upper limit was set to 50.0 for both $A$ and $I$. All simulations were coded in C. Results of three-dimensional simulations were visualized using OpenGL.

\section{Volume evaluation of the enclosed extracellular space}

497 To reduce computation time, we considered a cell shape with z-axis symmetry so that the 498 simulations can be run in the quasi 3-dimensional space with the $\mathrm{z}$ axis-symmetric 499 coordinate (i.e., on a $z-r$ plane). In this coordinate, $\nabla^{2}$ and $\nabla \cdot \vec{v}$ were replaced by $500 \quad \nabla^{2}=r^{-1} \frac{\partial}{\partial r}\left(r \frac{\partial}{\partial r}\right)+\frac{\partial}{\partial z}$ and $\nabla \cdot \vec{v}=\frac{1}{r} \frac{\partial}{\partial r}\left(r v_{r}\right)+\frac{\partial}{\partial z} v_{z}$. The Neumann boundary condition $501 \partial_{r} \phi=\partial_{r} A=\partial_{r} I=0$ are applied at the boundary $r=0$, whereas Dirichlet boundary 502 condition $\phi=A=I=0$ were applied for boundaries at $z=0, L_{z}$ and $r=L_{r}$, where $503 L_{z}, L_{r}$ are the axial length of the system. The analysis consisted of two parts (fig. S1G, $504 H$ ): (1) scoring of the membrane enclosing events (i.e., whether or not the region with $505 \phi=0$ that is enclosed by $\phi=1$ exists), and (2) estimating the enclosed volume at the 506 time of cup closure. For the first part, for each simulation time step, the number of 507 transition from $\phi=0$ to $\phi=1$ (red circles in fig. S1G) was counted along the line $r=$ $508 \Delta r$ from $(\Delta r, \mathrm{Lz})$ to $(\Delta r, 0)$. By definition, an enclosed region is present when this 509 number is 4 (fig. S1G, right panel) otherwise no closure (fig. S1G, left panel). The 510 enclosed volume was estimated at the time of closure by integrating the cross-sectional 511 disk (fig. S1 $H$, left panel) or disk with a hole at the center (fig. S1 $H$, right panel) at 
512 constant $z$ within $z_{b} \leq z \leq z_{t}$, where $z_{t}$ and $z_{b}$ are the first and second point at

513 which $\phi$ changed from $\phi=0$ to 1 (fig. $\mathrm{S} 1 H$ ).

514

515

516

$517 \quad$ Acknowledgments

518 The authors thank Shuji Ishihara, Tetsuya Hiraiwa and Chikara Furusawa for helpful

519 discussions. This work was supported by Japan Society for Promotion of Science (JSPS)

520 Grant-in-Aid for Young Scientists JP18K13514 to NS, Japan Science and Technology

521 Agency (JST) CREST JPMJCR1923, MEXT KAKENHI JP19H05801 to SS and in part

522 by Joint Research by Exploratory Research Center on Life and Living Systems

523 (ExCELLS) Grant 18-204, MEXT KAKENHI JP19H05416, JP18H04759 and

524 JP16H01442; JSPS KAKENHI JP17H01812 and JP15KT0076 (to S.S.).

525

526

527

Author Contributions

528 NS and SS conceived the work. NS planned the project, formulated the model, wrote 529 and run the programs and performed all analysis. SS oversaw the project, supervised 530 the analysis and contributed to the interpretation of the results. NS and SS wrote the 531 manuscript.

532

533

534 References

535

536

537 1. J. S. King, R. R. Kay, The origins and evolution of macropinocytosis. Philos.

$538 \quad$ Trans. R. Soc. B Biol. Sci. 374 (2019).

539 2. U. Hacker, R. Albrecht, M. Maniak, Fluid-phase uptake by macropinocytosis in 540 dictyostelium. J. Cell Sci. 110, 105-112 (1997).

541 3. C. Commisso, et al., Macropinocytosis of protein is an amino acid supply route in Ras-transformed cells. Nature 497, 633-637 (2013). 
543 4. J. J. Kamphorst, et al., Human pancreatic cancer tumors are nutrient poor and tumor cells actively scavenge extracellular protein. Cancer Res. 75, 544-553 (2015).

546 5. C. C. Norbury, Drinking a lot is good for dendritic cells. Immunology 117, 443$547 \quad 451(2006)$.

548 6. S. Yoshida, A. D. Hoppe, N. Araki, J. A. Swanson, Sequential signaling in plasma-membrane domains during macropinosome formation in macrophages. $J$.

550 Cell Sci. 122, 3250-3261 (2009).

7. S. BoseDasgupta, J. Pieters, Inflammatory Stimuli Reprogram Macrophage Phagocytosis to Macropinocytosis for the Rapid Elimination of Pathogens. PLoS Pathog. 10 (2014).

555

8. N. D. Condon, et al., Macropinosome formation by tent pole ruffling in macrophages. J. Cell Biol. 217, 3873-3885 (2018).

9. H. Kabayama, et al., Syntaxin 1B suppresses macropinocytosis and semaphorin 3A-induced growth cone collapse. J. Neurosci. 31, 7357-7364 (2011).

10. J. Mercer, A. Helenius, Virus entry by macropinocytosis. Nat. Cell Biol. 11, 510-

560 11. J. J. Yerbury, Protein aggregates stimulate macropinocytosis facilitating their propagation. Prion 10, 119-126 (2016).

12. L. J. Hewlett, A. R. Prescott, C. Watts, The coated pit and macropinocytic pathways serve distinct endosome populations. J. Cell Biol. 124, 689-703 (1994).

13. J. A. Swanson, Shaping cups into phagosomes and macropinosomes. Nat. Rev. Mol. Cell Biol. 9, 639-649 (2008).

14. M. Herant, V. Heinrich, M. Dembo, Mechanics of neurophil phagocytosis: Experiments and quantitative models. J. Cell Sci. 119, 1903-1913 (2006). approaches to phagocytosis. Reports Prog. Phys. 80 (2017).

16. C. M. Buckley, J. S. King, Drinking problems: mechanisms of macropinosome formation and maturation. FEBS J. 284, 3778-3790 (2017).

573 17. O. D. Weiner, W. A. Marganski, L. F. Wu, S. J. Altschuler, M. W. Kirschner, An actin-based wave generator organizes cell motility. PLoS Biol. 5, 2053-2063 (2007). 
576 18. D. Taniguchi, et al., Phase geometries of two-dimensional excitable waves govern self-organized morphodynamics of amoeboid cells. Proc. Natl. Acad. Sci. U. S. A. 110, 5016-21 (2013).

19. E. Bernitt, H. G. Döbereiner, N. S. Gov, A. Yochelis, Fronts and waves of actin polymerization in a bistability-based mechanism of circular dorsal ruffles. Nat. Commun. 8 (2017).

582

20. S. Flemming, F. Font, S. Alonso, C. Beta, How cortical waves drive fission of motile cells. Proc. Natl. Acad. Sci., 201912428 (2020).

584

21. T. Bretschneider, et al., Dynamic Actin Patterns and Arp2/3 Assembly at the Substrate-Attached Surface of Motile Cells. Curr. Biol. 14, 1-10 (2004).

586

22. T. Itoh, J. Hasegawa, Mechanistic insights into the regulation of circular dorsal

587 ruffle formation. J. Biochem. 153, 21-29 (2013).

588

23. M. Gerhardt, et al., Actin and PIP3 waves in giant cells reveal the inherent length scale of an excited state. J. Cell Sci. 127, 4507-4517 (2014).

24. D. M. Veltman, et al., A plasma membrane template for macropinocytic cups. Elife 5, 24 (2016).

592

25. S. Najem, M. Grant, A phase field model for neural cell chemotropism. Epl 102,

593 $1-4(2013)$.

594 26. A. Moure, H. Gomez, Computational model for amoeboid motion: Coupling membrane and cytosol dynamics. Phys. Rev. E 94, 1-9 (2016).

27. D. Shao, W.-J. Rappel, H. Levine, Computational Model for Cell Morphodynamics. Phys. Rev. Lett. 105, 108104 (2010).

28. D. Shao, H. Levine, W. J. Rappel, Coupling actin flow, adhesion, and morphology in a computational cell motility model. Proc. Natl. Acad. Sci. U. S. A. 109, 6851-6856 (2012).

29. J. Lee, Insights into cell motility provided by the iterative use of mathematical modeling and experimentation. AIMS Biophys. 5, 97-124 (2018).

603 30. E. Tjhung, A. Tiribocchi, D. Marenduzzo, M. E. Cates, A minimal physical model captures the shapes of crawling cells. Nat. Commun. 6, 1-9 (2015).

605 31. S. E. J. Campbell, P. Bagchi, E. J. Campbell, As featured in : in the presence of obstacles $\dagger(2018)$ https:/doi.org/10.1039/c8sm00457a. 
607 32. M. D. Rueda-Contreras, J. R. Romero-Arias, J. L. Aragón, R. A. Barrio,

608

609

610

611

612

613

614

615

616

617

618

619

620

621

622

623

624

625

626

627

628

629

630

631

632

633

634

635

636

637

638 Curvature-driven spatial patterns in growing 3D domains: A mechanochemical model for phyllotaxis. PLoS One 13, 1-23 (2018).

33. A. Karma, W.-J. Rappel, Quantitative phase-field modeling of dendritic growth in two and three dimensions. Phys. Rev. E 57, 4323-4349 (1998).

34. C. Beckermann, H.-J. Diepers, I. Steinbach, A. Karma, X. Tong, Modeling Melt Convection in Phase-Field Simulations of Solidificatio. J. Comput. Phys. 154, 468-496 (1999).

35. J. S. Lowengrub, A. Rätz, A. Voigt, Phase-field modeling of the dynamics of multicomponent vesicles: Spinodal decomposition, coarsening, budding, and fission. Phys. Rev. E - Stat. Nonlinear, Soft Matter Phys. 79, 1-13 (2009).

36. M. Sadeghi, F. Noé, Large-scale simulation of biomembranes incorporating realistic kinetics into coarse-grained models. Nat. Commun. 11, 2951 (2020).

37. Y. Mori, A. Jilkine, L. Edelstein-Keshet, Wave-pinning and cell polarity from a bistable reaction-diffusion system. Biophys. J. 94, 3684-3697 (2008).

38. R. Diegmiller, H. Montanelli, C. B. Muratov, S. Y. Shvartsman, Spherical Caps in Cell Polarization. Biophys. J. 115, 26-30 (2018).

39. O. Hoeller, et al., Two distinct functions for PI3-kinases in macropinocytosis. $J$. Cell Sci. 126, 4296-4307 (2013).

40. D. M. Veltman, M. G. Lemieux, D. A. Knecht, R. H. Insall, PIP3-dependent macropinocytosis is incompatible with chemotaxis. J. Cell Biol. 204, 497-505 (2014).

41. T. D. Williams, P. I. Paschke, R. R. Kay, Function of small GTPases in Dictyostelium macropinocytosis. Philos. Trans. R. Soc. B Biol. Sci. 374 (2019).

42. B. Álvarez-González, et al., Three-dimensional balance of cortical tension and axial contractility enables fast amoeboid migration. Biophys. J. 108, 821-832 (2015).

43. V. C. Abraham, V. Krishnamurthi, D. Lansing Taylor, F. Lanni, The actin-based nanomachine at the leading edge of migrating cells. Biophys. J. 77, 1721-1732 (1999).

44. H. Inaba, K. Yoda, H. Adachi, The F-actin-binding RapGEF GflB is required for efficient macropinocytosis in Dictyostelium. J. Cell Sci. 130, 3158-3172 (2017). 
639 45. G. Bloomfield, et al., Neurofibromin controls macropinocytosis and phagocytosis

640

641

642

643

644

645

646

647

648

649

650

651

652

653

654

655

656

657

658

659

660

661

662

663

664

665

666

667

668

669

670

671 in Dictyostelium. Elife 2015, 1-25 (2015).

46. G. Gerisch, B. Schroth-Diez, A. Müller-Taubenberger, M. Ecke, PIP3 waves and PTEN dynamics in the emergence of cell polarity. Biophys. J. 103, 1170-1178 (2012).

47. M. Jasnin, et al., The Architecture of Traveling Actin Waves Revealed by CryoElectron Tomography. Structure 27, 1211-1223.e5 (2019).

48. G. Bloomfield, R. R. Kay, Uses and abuses of macropinocytosis. J. Cell Sci. 129, 2697-2705 (2016).

49. M. Kaksonen, A. Roux, Mechanisms of clathrin-mediated endocytosis. Nat. Rev. Mol. Cell Biol. 19, 313-326 (2018).

50. A. Frost, et al., Structural Basis of Membrane Invagination by F-BAR Domains. Cell 132, 807-817 (2008).

51. H. Noguchi, Membrane tubule formation by banana-shaped proteins with or without transient network structure. Sci. Rep. 6, 1-8 (2016).

52. Y. Miao, et al., Wave patterns organize cellular protrusions and control cortical dynamics. 1-20 (2019).

53. S. Funamoto, R. Meili, S. Lee, L. Parry, R. A. Firtel, Spatial and temporal regulation of 3-phosphoinositides by PI 3-kinase and PTEN mediates chemotaxis. Cell 109, 611-623 (2002).

54. A. T. Sasaki, et al., G protein-independent Ras/PI3K/F-actin circuit regulates basic cell motility. J. Cell Biol. 178, 185-191 (2007).

55. D. Bar-Sagi, J. R. Feramisco, Induction of membrane ruffling and fluid-phase pinocytosis in quiescent fibroblasts by ras proteins. Science (80-. ). 233, 10611068 (1986).

56. J. R. Chubb, A. Wilkins, G. M. Thomas, R. H. Insall, The Dictyostelium RasS protein is required for macropinocytosis, phagocytosis and the control of cell movement. J. Cell Sci. 113, 709-719 (2000).

57. T. D. Williams, S. Y. Peak-Chew, P. Paschke, R. R. Kay, Akt and SGK protein kinases are required for efficient feeding by macropinocytosis. J. Cell Sci. 132 (2019).

58. J. Loh, et al., An acute decrease in plasma membrane tension induces macropinocytosis via PLD2 activation. J. Cell Sci. 132 (2019). 
672 59. T. A. Burke, et al., Homeostatic actin cytoskeleton networks are regulated by

673

674

675

676

677

678

679

680

681

682

683

684

685

686

687

688

689

690

691

692

693

694

695

696

697

698

699

700

701

702

703

704 assembly factor competition for monomers. Curr. Biol. 24, 579-585 (2014).

60. A. J. Lomakin, et al., Competition for actin between two distinct F-actin networks defines a bistable switch for cell polarization. Nat. Cell Biol. 17, 14351445 (2015).

61. C. Suarez, D. R. Kovar, Internetwork competition for monomers governs actin cytoskeleton organization. Nat. Rev. Mol. Cell Biol. 17, 799-810 (2016).

62. M. F. Carlier, S. Shekhar, Global treadmilling coordinates actin turnover and controls the size of actin networks. Nat. Rev. Mol. Cell Biol. 18, 389-401 (2017).

63. A. Antkowiak, et al., Sizes of actin networks sharing a common environment are determined by the relative rates of assembly. PLoS Biol. 17, 1-25 (2019).

64. P. Bleicher, A. Sciortino, A. R. Bausch, The dynamics of actin network turnover is self-organized by a growth-depletion feedback. Sci. Rep. 10, 1-11 (2020).

65. J. Helenius, M. Ecke, D. J. Müller, G. Gerisch, Oscillatory Switches of DorsoVentral Polarity in Cells Confined between Two Surfaces. Biophys. J. 115, 150162 (2018).

66. T. Bretschneider, et al., The three-dimensional dynamics of actin waves, a model of cytoskeletal self-organization. Biophys. J. 96, 2888-2900 (2009).

67. B. L. Goode, et al., Coronin promotes the rapid assembly and cross-linking of actin filaments and may link the actin and microtubule cytoskeletons in yeast. $J$. Cell Biol. 144, 83-98 (1999).

68. E. L. De Hostos, The coronin family of actin-associated proteins. Trends Cell Biol. 9, 345-350 (1999).

69. A. De Baey, A. Lanzavecchia, The role of aquaporins in dendritic cell macropinocytosis. J. Exp. Med. 191, 743-747 (2000).

70. M. Chabaud, et al., Cell migration and antigen capture are antagonistic processes coupled by myosin II in dendritic cells. Nat. Commun. 6, 1-16 (2015).

71. J. Dai, H. P. Ting-Beall, R. M. Hochmuth, M. P. Sheetz, M. A. Titus, Myosin I contributes to the generation of resting cortical tension. Biophys. J. 77, $1168-$ 1176 (1999).

72. H. Brzeska, H. Koech, K. J. Pridham, E. D. Korn, M. A. Titus, Selective localization of myosin-I proteins in macropinosomes and actin waves. Cytoskeleton 73, 68-82 (2016). 
bioRxiv preprint doi: https://doi.org/10.1101/2020.06.22.165027; this version posted June 23, 2020. The copyright holder for this preprint (which was not certified by peer review) is the author/funder, who has granted bioRxiv a license to display the preprint in perpetuity. It is made available under aCC-BY-NC-ND 4.0 International license.

705 73. N. Araki, T. Hatae, T. Yamada, S. Hirohashi, Actinin-4 is preferentially involved 706 in circular ruffling and macropinocytosis in mouse macrophages: Analysis by

707 fluorescence ratio imaging. J. Cell Sci. 113, 3329-3340 (2000).

708

709

710 
714 Fig.1: Macropinocytic cup formation and the model schematics. $(A)$ Time sequence 715 of macropinocytic cup formation (left to right). A micrometer-scale membrane domain; 716 “active patch" (red) enriched in small GTPases and phosphoinositides grows and expands

717 in the plasma membrane. The Scar/WAVE complex is localized at the edge of a patch 718 (black) (24). (B) Phase field $\phi$ defines the state of position $x$ in space; occupied $(\phi=1)$ 719 or vacant $(\phi=0)$. An auxiliary variable $\psi$ is introduced to delineate the border $(\psi=$ 720 1) i.e. the plasma membrane and the rest of the space $(\psi=0)$. (C) The schematic diagram 721 of the model reaction. $A$ and $B$ are active and inactive form of an active patch factor, 722 respectively. $I$ is a factor that suppresses the positive feedback amplification of $A$ at 723 the membrane. (D) The spatial profile of protruding force $F_{\text {poly }}$ (Eq.(7)) is determined 724 by the distribution of $A$. A representative data for a 2D-planar membrane $\left(K_{1}=0.005\right.$, $725 \quad K_{2}=0.25$ and $\left.n_{h}=3\right)$.

Fig.2 Membrane protrusion at the edge of an active patch is sufficient for the formation of the basic cup-like structure and its closure. Simulation results: $(A)$ a representative time course of the numerical simulations $\left(F / \eta=4.0, a_{t}=2.8\right)$. The active patch (red; $A \psi>0$ ) and the membrane (green; $\psi>0$ ) shown as merged RGB images; birds-eye view (upper panels) and as cross sections along the median plane (lower panels). Asterisks indicate cup closure. $(B)$ Phase diagram of the cup dynamics. Color bars indicate the volume of enclosure normalized by the cell size (blue to yellow). The cutoff volume for successful cup closure was set to $<10^{-5}$ (black). Averages of six independent simulation runs (three of each for $r_{\text {init }}=1.0 \mu \mathrm{m}$ and $1.5 \mu \mathrm{m}$ ) are shown.

737 Phase I and II: enclosure in all or part of the six trials, respectively. Phase III: repetitive 738 cup formation. Phase IV: cup closure failed in all simulations runs. The red dashed line 739 is the estimated minimal force $F / \eta=2.0$ required for protrusion. Parameter sets in $(A)$ 740 and $(C-G)$ are indicated in the diagram. $(C-G)$ Representative time course for $(C)$ $741 \quad F / \eta=5.2, a_{\mathrm{t}}=2.6(D) F / \eta=1.6, a_{\mathrm{t}}=2.8,(E) F / \eta=3.2, a_{\mathrm{t}}=2.5,(F) F / \eta=2.8$, 
$a_{\mathrm{t}}=2.8,(G) F / \eta=2.4, a_{\mathrm{t}}=2.7$. Other parameters: $\tau=10, D_{a}=0.1, \alpha=1.0, \varepsilon=$ $0.8, M_{V}=5.0, \beta=100.0, \theta=0.105, \eta=0.5$.

Fig.3: Complex cell morphologies result from multiple stochastic patch initiation.

$(A, B)$ Representative snapshots from independent simulations with the same parameter sets as Fig. $2 A$ and $F(I=0)$. Top overhead view (upper panels) and the midline cross section (lower panels) with merged RGB images (Green: cell membrane $(\psi>0)$. Red: active patches $(A \psi>0)$. Noise parameters: $\sigma=8.0, d=15.0, \lambda=3 \times 10^{-5}$.

Fig.4: Presence of an inhibitor gives rise to cup splitting dynamics. $(A)$ Phase diagram of chemical reaction Eq.(2) decoupled from deformation dynamics in the presence of inhibitor kinetics (Eq.(3)) for $k_{1}=0.088$. Depending on $k_{2}$, the system is bi-stable $\left(k_{2}>\right.$ 1) (red region) or excitable $\left(k_{2}<1\right)$ (pink region). A single fixed point $A=0$ for $a_{t}<$ $2 \sqrt{k_{1} / k_{2}}$ (white region) and three fixed points $A=0$, and $A^{ \pm}=(B \pm$ $\left.\sqrt{B^{2}-4 k_{1} / k_{2}}\right) /\left(2 k_{1} / k_{2}\right)$ for $a_{t}>2 \sqrt{k_{1} / k_{2}}$ (pink and red regions). $A=0$; stable. $A^{-}$; unstable. $A^{+}$; stable $\left(k_{2}>1\right)$ or unstable $\left(k_{2}<1\right)$. (B) Null-clines in the excitable regime for $B>2 \sqrt{k_{1} / k_{2}}$ (left panel) and $B<2 \sqrt{k_{1} / k_{2}}$ (right panel). Fixed points (Filled circle: stable. Open circle: unstable). Excitatory trajectories (red arrows) invoked by small perturbation to $A=0 .(C, D)$ Representative dynamics $\left(a_{\mathrm{t}}=1.985\right.$, $k_{1}=0.088, k_{2}=0.54, D_{a}=0.085$ and $\left.D_{i}=0.11\right)$ on a fixed spherical field $(C)$ and deforming membrane $(D)\left(\tau=7.0, F=3.7, K_{1}=0.01, K_{2}=0.1\right.$ and $\left.n_{h}=5\right)$. (E, $F) \quad$ Representative dynamics $\left(a_{\mathrm{t}}=1.94, k_{1}=0.088, k_{2}=0.54, D_{a}=0.26\right.$ and $\left.D_{i}=0.87\right)$ on a fixed spherical field $(E)$ and deforming membrane $(F)(\tau=20.0, F=$ 3.0, $K_{1}=0.086, K_{2}=1.8$ and $n_{h}=3$ ). Other parameters are same as in Fig. 2. 
bioRxiv preprint doi: https://doi.org/10.1101/2020.06.22.165027; this version posted June 23, 2020. The copyright holder for this preprint (which was not certified by peer review) is the author/funder, who has granted bioRxiv a license to display the preprint in perpetuity. It is

Fig. 1 made available under aCC-BY-NC-ND 4.0 International license.

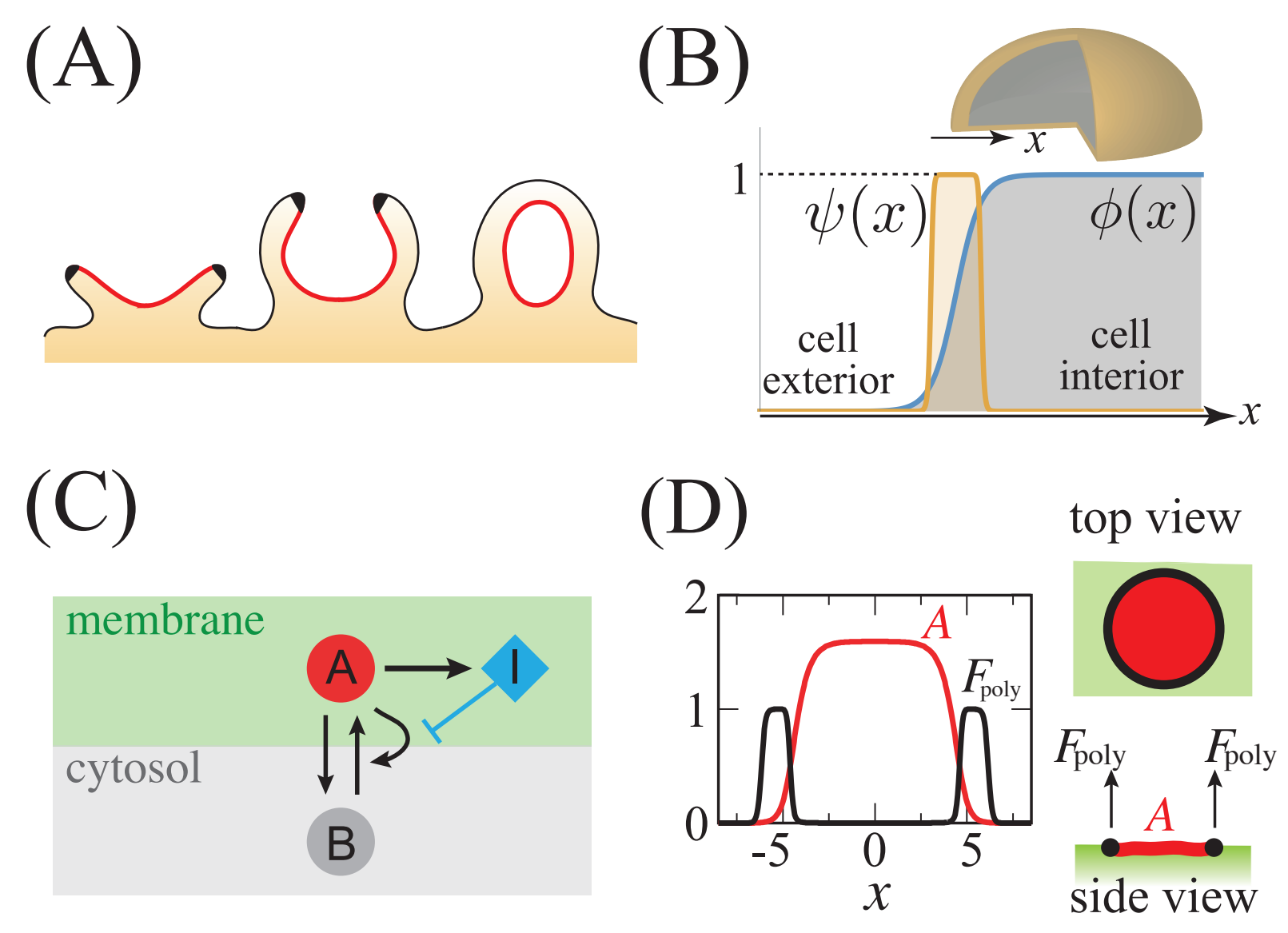


bioRxiv preprint doi: https://doi.org/10.1101/2020.06.22.165027; this version posted June 23, 2020. The copyright holder for this preprint (which was not certified by peer review) is the author/funder, who has granted bioRxiv a license to display the preprint in perpetuity. It is made available under aCC-BY-NC-ND 4.0 International license.

Fig. 2

$(\mathrm{A})_{t=4}$
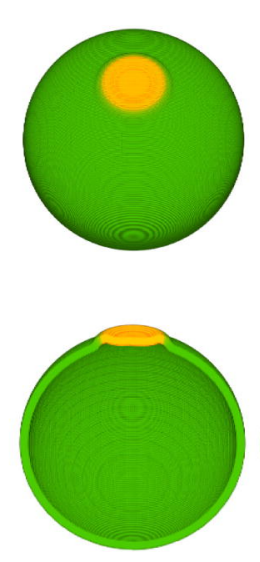

(C)

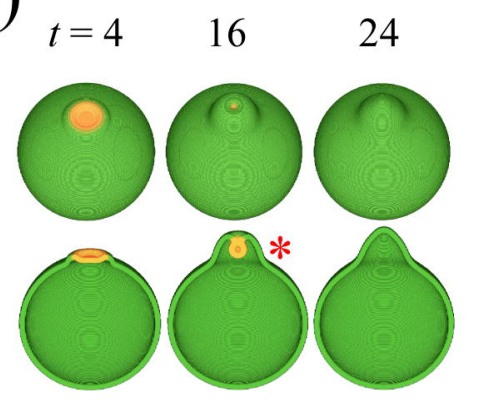

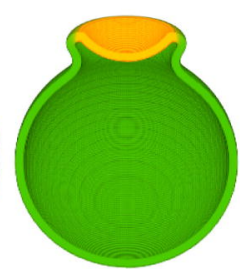

44
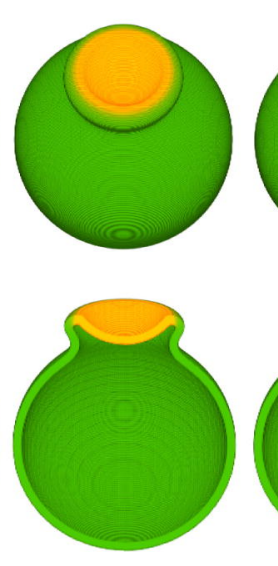
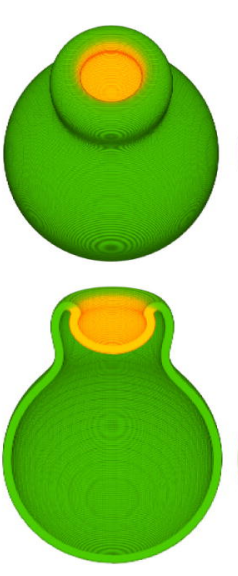

(D) ${ }_{t=4}$
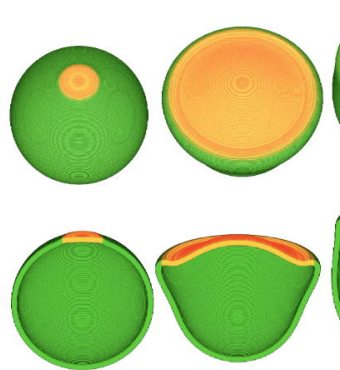

(G)

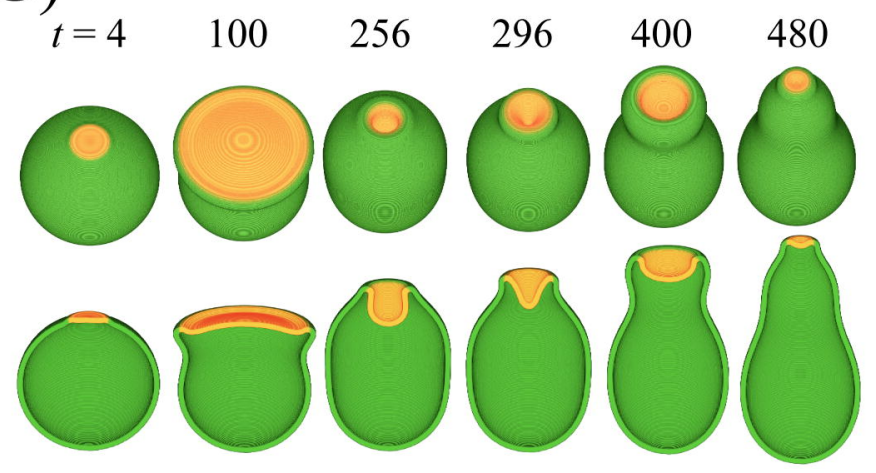

(B)

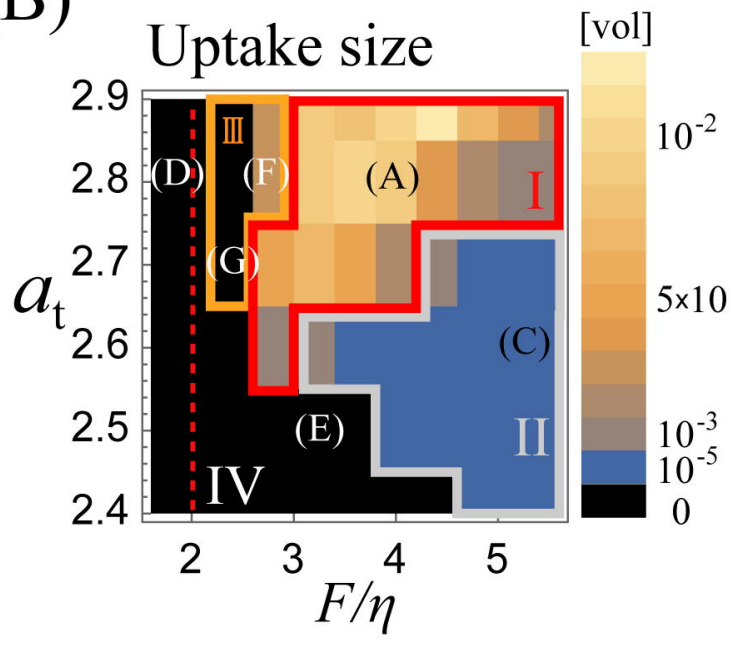

$(\mathrm{E})$

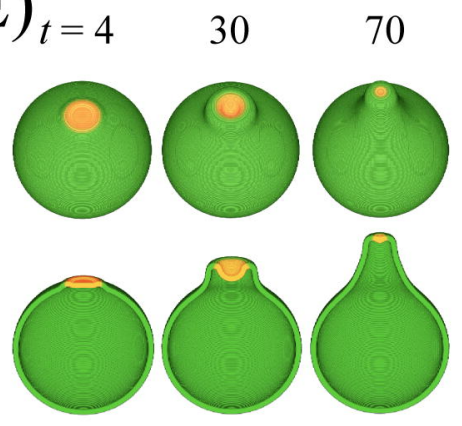

$10^{-2}$

$5 \times 10$

$10^{-3}$

$10^{-5}$
65
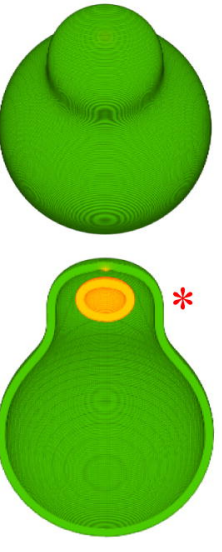

690
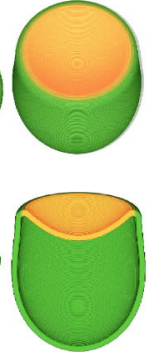

(F)

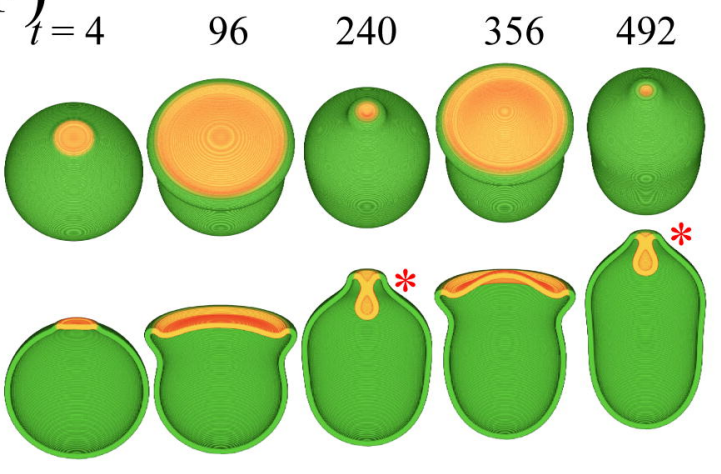


Fig. 3

(A) $a_{\mathrm{t}}=2.8 \quad F / \eta=4.0$

top
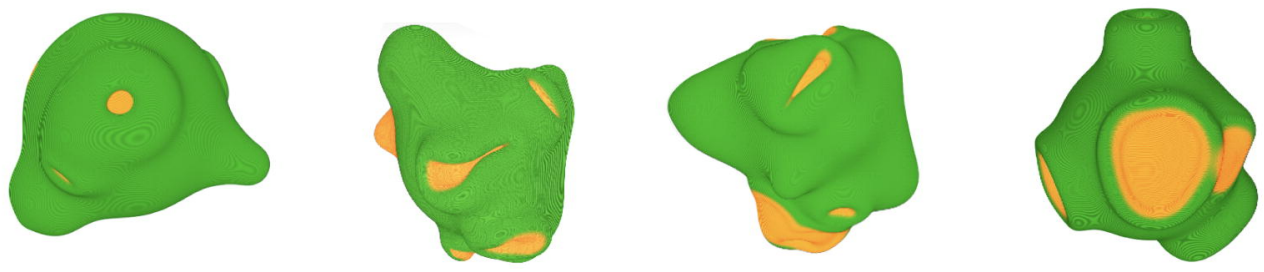

cross-
section
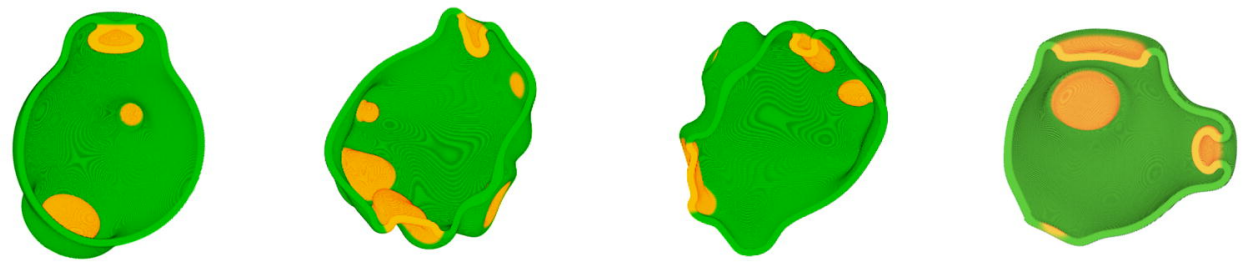

(B) $a_{\mathrm{t}}=2.8 \quad F / \eta=2.8$

top
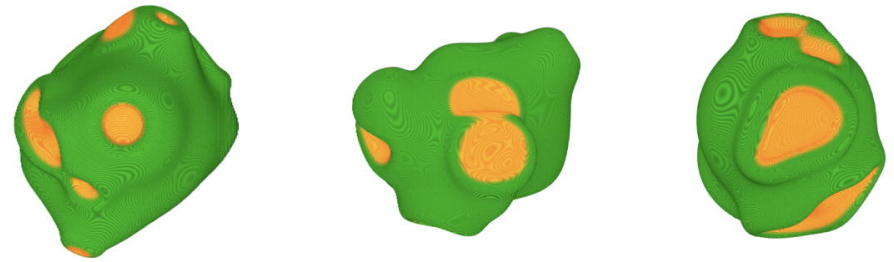

crosssection
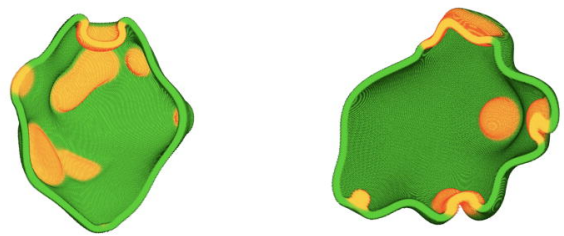
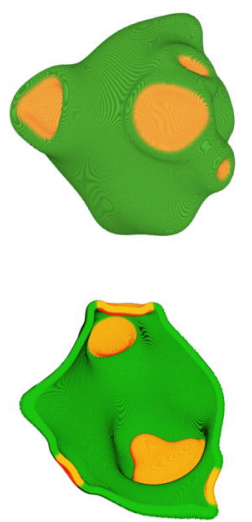
FigRiv preprint doi: https://doi.org/10.1101/2020.06.22.165027; this version posted June 23, 2020. The copyright holder for this preprint $\mathrm{F} 1 \mathrm{~g}$. (4ich was not certified by peer review) is the author/funder, who has granted bioRxiv a license to display the preprint in perpetuity. It is made available under aCC-BY-NC-ND 4.0 International license.

(A)

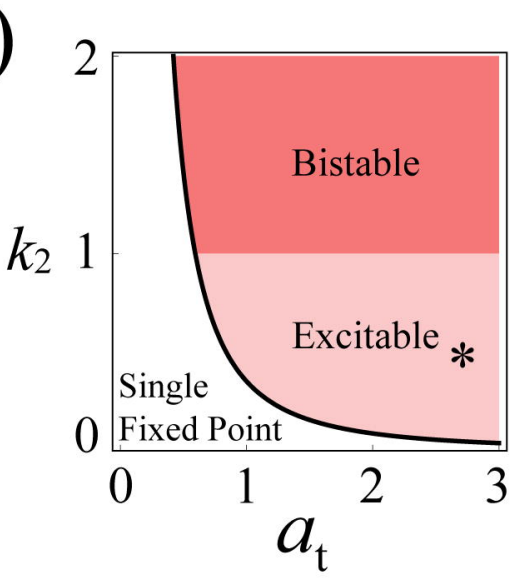

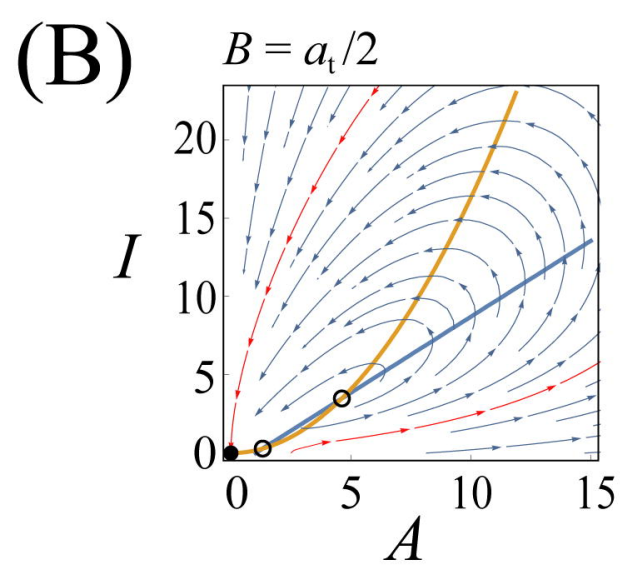

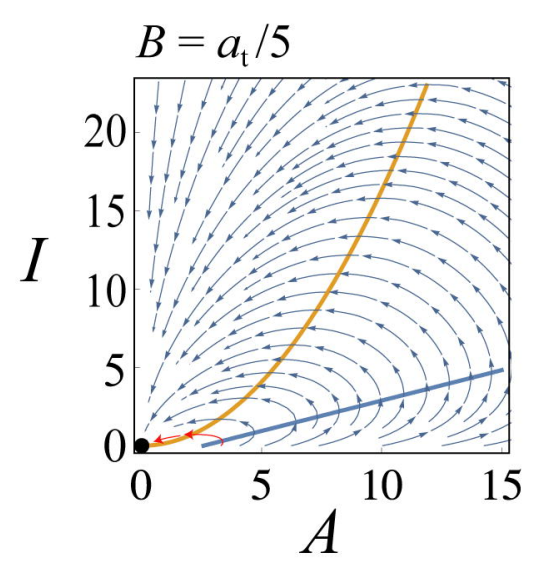

(C)

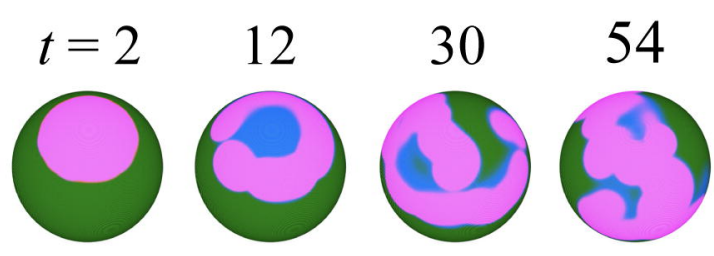

(D)
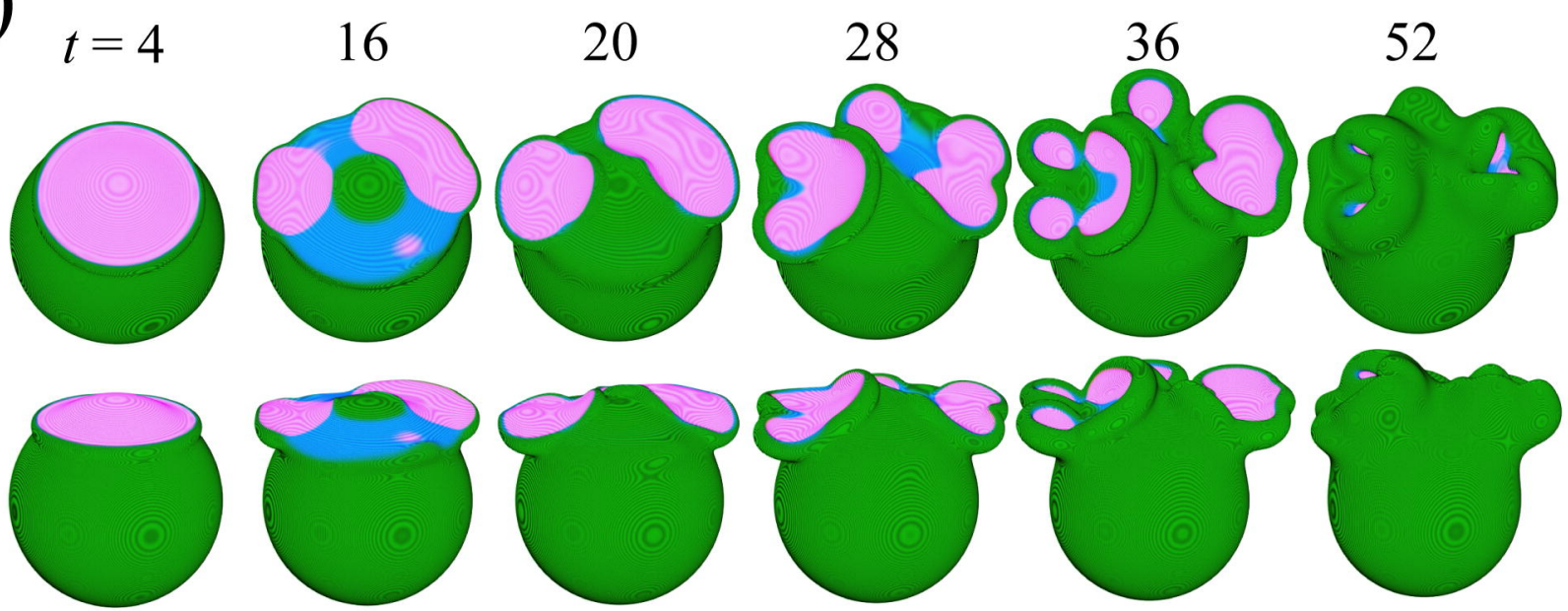

60
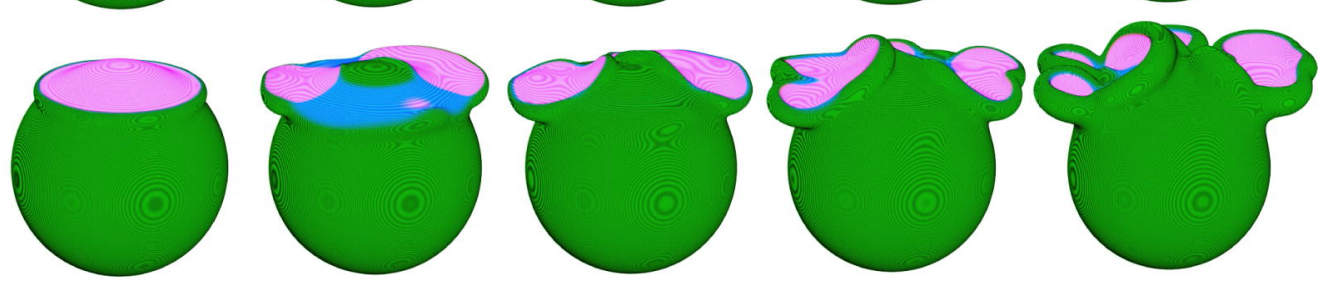

(E) $t=2 \quad 18 \quad 38 \quad 184$

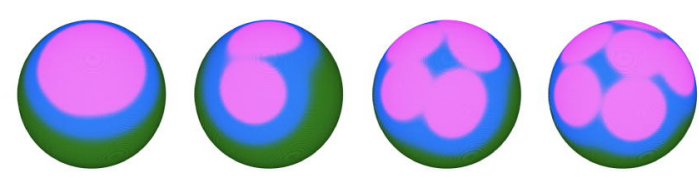

(F)
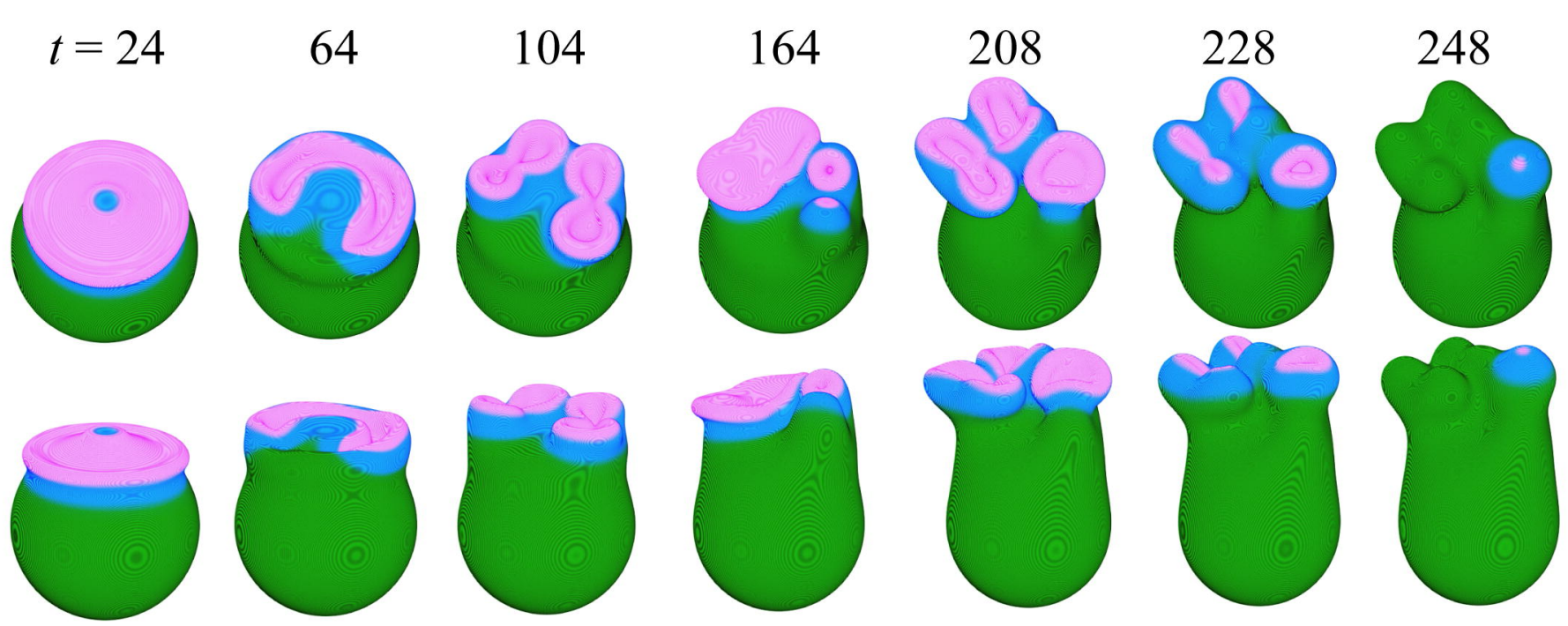